\title{
Self-management reduces both short- and long-term hospitalisation in COPD
}

\author{
M-A. Gadoury*, K. Schwartzman*, M. Rouleau\# , F. Maltais ${ }^{\ddagger}$, M. Julien+, \\ A. Beaupré ${ }^{\S}$, P. Renzi ${ }^{\dagger}$, R. Bégin**, D. Nault* and J. Bourbeau*, \\ for the Chronic Obstructive Pulmonary Disease axis of the Respiratory Health \\ Network, Fonds de la recherche en santé du Québec (FRSQ)
}

ABSTRACT: The aim of the present study was to assess the long-term impact on hospitalisation of a self-management programme for chronic obstructive pulmonary disease (COPD) patients.

A multicentre, randomised clinical trial was carried out involving 191 COPD patients from seven hospitals. Patients who had one or more hospitalisations in the year preceding study enrolment were assigned to a self-management programme "Living Well with COPD" Hospitalisations from all causes were the primary outcome and were documented from the provincial hospitalisation database; emergency visits were recorded from the provincial health insurance database.

Most patients were elderly, not highly educated, had advanced COPD (reflected by a mean forced expiratory volume in one second of $1 \mathrm{~L}$ ), and almost half reported a dyspnoea score of 5/5 (modified Medical Research Council). At 2 years, there was a statistically significant and clinically relevant reduction in all-cause hospitalisations of $26.9 \%$ and in all-cause emergency visits of $\mathbf{2 1 . 1 \%}$ in the intervention group as compared to the standard-care group. After adjustment for the self-management intervention effect, the predictive factors for reduced hospitalisations included younger age, sex (female), higher education, increased health status and exercise capacity.

In conclusion, in this study, patients with chronic obstructive pulmonary disease who received educational intervention with supervision and support based on disease-specific self-management maintained a significant reduction in hospitalisations after a 2-year period.

KEYWORDS: Chronic obstructive pulmonary disease, education, hospital utilisation, long-term care, self-management

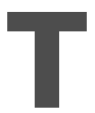

he burden and costs of chronic obstructive pulmonary disease (COPD) are important and are expected to grow in the near future $[1,2]$. COPD progresses through different stages, and has a major impact on functional abilities and quality of life [3-5]. The disease is also characterised by episodes of acute exacerbations that contribute to the deterioration of patients' health status $[4,5]$.

Acute exacerbations require frequent medical evaluation and increase the utilisation of health resources, especially in those patients with poor health status [6]. COPD patients often require emergency and hospital admissions [7, 8], which are the largest contributors to healthcare costs [9]. The Obstructive Lung Disease in Northern Sweden (OLIN) studies showed that hospitalisation costs account for $>65 \%$ of all COPD costs and up to $90 \%$ of the diseaserelated expenses of those with more severe disease [9].
Recently, a self-management programme specifically developed for COPD patients, "Living Well with $\mathrm{COPD}^{\mathrm{TM}}$ " (Boehringer Ingelheim), involving communication with a trained health professional over $1 \mathrm{yr}$, has been shown to reduce the utilisation of healthcare services and improve health status in the short term [8]. However, from this multicentre, randomised clinical trial, it remains unknown if the benefits of the programme, with respect to the reduction in hospitalisations and emergency department visits, were sustained beyond $1 \mathrm{yr}$.

The current study hypothesised that those patients who followed the self-management education programme, as compared to a group of patients on standard care, would maintain a lower rate of hospitalisation for $\geqslant 2$ yrs from programme administration.

\section{MATERIALS AND METHODS}

The design of and methods used in the present trial have been previously described [8].

\section{AFFILIATIONS}

*Respiratory Epidemiology and Clinical Research Unit, McGill University and Montreal Chest Institute of the Royal Victoria Hospital, McGill University Health Centre, and

+Hôpital du Sacré-Coeur

${ }^{\S}$ Hôpital Maisonneuve-Rosemont, and ${ }^{f}$ Hôpital Notre-Dame, Centres Hospitaliers Affiliés de I'Université, Montréal, and

\#Hôpital du St-Sacrement, Centre Universitaire Affilié de l'Université Laval, Québec,

"Hôpital Laval, Institut Universitaire de Cardiologie et de Pneumologie, Université Laval, Ste-Foy, and ${ }^{*}{ }^{*}$ Centre Hospitalier Universitaire de Sherbrooke, Sherbrooke,

Québec, Canada.

\section{CORRESPONDENCE}

J. Bourbeau, Respiratory Epidemiology and Clinical Research Unit, 3650 St Urbain Street, Montréal, Québec, H2X 2P4, Canada

Fax: 15148432083

E-mail: jean.bourbeau@mcgill.ca

Received:

August 092004

Accepted after revision:

July 182005

\section{SUPPORT STATEMENT}

This study was funded by the Fond de la recherche en santé du Québec (FRSQ) and by an unrestricted grant from Boehringer Ingelheim Canada. The industry sponsor was excluded from participation trial board policy, data management and final data analysis. J. Bourbeau, F. Maltais and K. Schwartzman are recipients of FRSQ research awards. 
However, the trial design, patient selection methods and study interventions are summarised as follows.

\section{Study design}

The present study is a follow-up to a multicentre, randomised clinical trial conducted over $1 \mathrm{yr}$ in 191 COPD patients from seven health centres. The intervention group followed a disease-specific self-management programme, "Living Well with COPD ${ }^{\mathrm{TM}}$ ", in addition to standard care. The comparison group received standard care alone. The current study includes 2 yrs of follow-up with two additional sources of information: the provincial health insurance and hospitalisation database records, which capture all physician encounters, and are coded by location and diagnosis; and all hospital separations.

\section{Patient selection}

Between February and July 1998, seven centres randomly assigned 191 patients to two treatment arms. All patients provided written informed consent, and each institutional research board approved the study. The Commission d'Accès à l'Information du Québec (Québec, Canada) approved the use of the administrative databases. Patients had to be aged $\geqslant 50$ yrs. Study entry was restricted to former or current smokers. Patients had to have a forced expiratory volume in one second (FEV1) $<70 \%$ predicted. Patients also had to have been hospitalised once or more for COPD exacerbation in the year preceding their enrolment into the study. Patients with asthma as a primary diagnosis, and those with major comorbidities, such as documented left ventricular failure and any terminal disease, were excluded. Finally, patients with dementia or uncontrolled psychiatric illness were also excluded.

\section{Study intervention}

Patients in the standard care and intervention groups continued to be managed by their usual physicians, and they continued to have free access to the universal health and drug insurance programmes administered by the Québec government. The intervention was a disease-specific self-management multi-component programme, "Living Well with $\mathrm{COPD}^{\mathrm{TM}}$ ", consisting of $\sim 1 \mathrm{~h}$ each week of skill-oriented teaching for 7-8 weeks. The education programme was originally developed at the Montreal Chest Institute (Montreal, Canada), in collaboration with the Respiratory Health Network of the Fonds de la Recherche en Santé du Québec (FRSQ) and Boehringer Ingelheim (Burlington, Canada). The programme included a patient workbook [8]: an action plan with a customised prescription for antibiotics and prednisone for when the patient had exacerbations. Finally, all intervention patients received advice on a simple home exercise programme that was not supervised, except for an initiation visit.

All patients except one complied with the first 7-8 weeks of the programme administration at home. During the 1-yr study, patients had monthly telephone follow-up assessments, and they could also contact the case manager during working hours. There was no formalised follow-up beyond the 1st yr of the study, and only two out of the seven participating centres continued to have access to a case manager.

\section{Assessment of outcomes}

The primary outcome was all-cause hospital admissions. Secondary outcomes included all-cause emergency visits. To assess hospitalisations and emergency visits, data collected during the clinical trial were linked to the hospitalisation (Med-Echo) and health insurance (Régie de l'Assurance Maladie du Québec (RAMQ)) databases. Access to these data was available for the year preceding enrolment in the trial, and for the 1st and 2nd yrs after patient entry in the study.

\section{Statistical analysis}

An intent-to-treat analysis was performed, including all randomised subjects, regardless of their adherence to the intervention. For the primary analysis, comparison of the rate of all-cause hospitalisations in the 2nd yr of follow-up was performed with the use of an unadjusted Poisson distribution test. The rate of hospitalisation was taken as the number of hospitalisations divided by the total time at risk. Total time at risk was taken as being equal to the time of follow-up minus the time of hospitalisations. This analysis was also performed to assess the treatment difference between the study groups in the rate of all-cause emergency room visits in the 2nd yr, as well as for the preceding year and the study year. Further analysis included Poisson regression modelling, in order to find the predictive capacity of a set of variables after adjustment for the self-management intervention. A Poisson distribution was used, since it best describes the number of hospitalisations. Finally, statistically significant variables were kept in the model in a backward selection process. Variables were eliminated manually when their $p$-value was $>0.05$ or if the explanatory power of the model was not improved by their inclusion. Reported p-values are two-tailed, without corrections for multiple comparisons.

\section{RESULTS}

\section{Study patients}

Figure 1 shows detailed information on enrolment, allocation to the study treatment, loss to follow-up and death. A total of

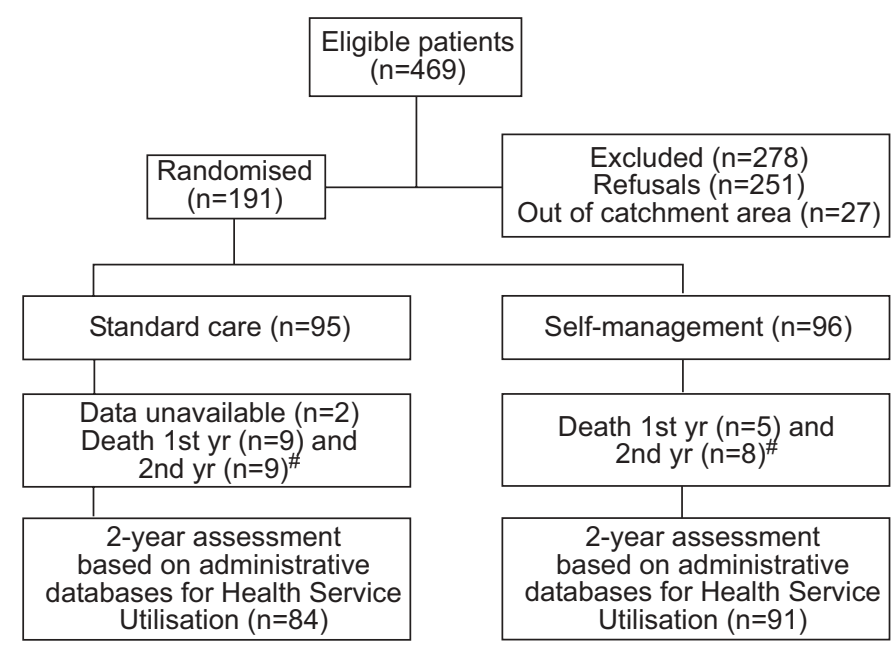

FIGURE 1. Trial profile based on hospitalisation, collected from a provincial

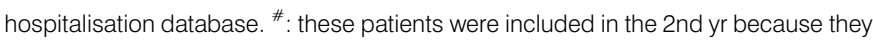
experienced emergency room visits or hospitalisations. 


\begin{tabular}{lcc} 
TABLE 1 & $\begin{array}{c}\text { Baseline characteristics of the study patients } \\
\text { Intervention group }\end{array}$ & Usual care group \\
\hline Subjects $\mathbf{n}$ & 96 & 95 \\
Age yrs & $69.4 \pm 6.5$ & $69.6 \pm 7.4$ \\
Sex male & $50(52)$ & $56(59)$ \\
Living alone & $46(48)$ & $40(42)$ \\
Education $\leqslant$ 12th grade & $79(82)$ & $73(77)$ \\
Current smoker & $24(25)$ & $25(26)$ \\
Smoking pack-yrs & $57.8 \pm 40.6$ & $56.1 \pm 31.3$ \\
FEV $\mathbf{L}$ & $0.98 \pm 0.31$ & $1.00 \pm 0.33$ \\
FEV $\mathbf{1}$ FVC ratio & $0.46 \pm 0.10$ & $0.45 \pm 0.12$ \\
Dyspnoea MRC $\mathbf{5 / 5}$ & $44(46)$ & $47(49)$ \\
6-min walk test $\mathbf{~ m}$ & $282 \pm 91$ & $280 \pm 90$ \\
SGRQ\# & $54.1 \pm 16.6$ & $55.7 \pm 15.7$
\end{tabular}

Data are presented as mean \pm SD or $n(\%)$, unless otherwise stated. FEV1: forced expiratory volume in one second; FVC: forced vital capacity; MRC: Medical Research Council; SGRQ: St George's Respiratory Questionnaire. \#: lower score on the questionnaire indicates better health status.

469 patients were eligible; however, 251 refused to participate in the study mainly because of the burden of the 1st $\mathrm{yr}$ evaluation and 27 because they lived out of the catchment areas. Those who refused were similar to the study patients with respect to sex, age and disease severity. Of those eligible, 191 subjects were randomised, 95 to standard care and 96 to the self-management education programme. At the end of the 2nd yr of follow-up, data were available for 75 patients in the standard-care group (two subjects were lost to follow-up, nine patients died in the 1st yr and nine in the 2nd yr) and 83 patients following the self-management programme (five patients died in the 1st yr and eight in the 2nd yr).

Table 1 presents the patient baseline characteristics in each treatment group. Patients in both groups had similar sociodemographic and clinical characteristics. Patients were elderly, smokers or ex-smokers and not highly educated. Patients in both groups had similar disease severity according to FEV1, dyspnoea and exercise capacity.

\section{Hospital admissions}

Table 2 shows the comparison of all-cause hospitalisations between the two study groups. In the self-management group, there was a reduction in the rate of all-cause hospitalisations in the 1st yr of the study as compared to the year preceding enrolment. This reduction was still present during the 2nd yr of follow-up, although to a lesser degree. The standard-care group experienced a slight increase in all-cause hospitalisations in the 1st yr as compared to the year preceding enrolment. This remained unchanged at 2 yrs, follow-up. There was a statistically significant reduction in the rate of allcause hospitalisations in the self-management group as compared to the standard care group during the 1st yr $\left(-0.7\right.$ hospitalisations patient-yr ${ }^{-1} \quad(95 \%$ confidence interval $-0.95--0.46))$, as well as during the 2 nd yr of follow-up $(-0.44$ hospitalisations $\cdot$ patient- $\left.\mathrm{yr}^{-1}(-0.68--0.21)\right)$.

\section{Emergency room visits}

Table 3 shows the comparison of all-cause emergency room visits between the two study groups. In the self-management group, there was a reduction in the rate of all-cause emergency room visits in the 1st yr of the study as compared to the year preceding enrolment. This reduction was still present during the 2nd yr of follow-up, although to a lesser degree. The standard-care group experienced a slight increase in the rate of all-cause emergency room visits in the 1st yr as compared to the year preceding enrolment. This remained unchanged during the 2nd yr of follow-up. There was a statistically significant reduction in the rate of all-cause emergency room visits in the self-management group as compared to the standard-care group during the 1st yr (-1.3 emergency room visits patient- $\left.\mathrm{yr}^{-1}(-1.18--1.42)\right)$, as well as during the 2 nd $\mathrm{yr}$ of follow-up (-0.7 emergency room visits $\cdot$ patient- $\mathrm{yr}^{-1}$ $(-0.58--0.82))$.

\section{Predicting factors of hospitalisation}

The results of the Poisson regression model analysis are presented in table 4. Predictive factors of reduced hospitalisations after adjustment for the self-management intervention included sex (female), increased walking distance and higher education. Predictive factors of increased hospitalisations included older age and reduced health status.

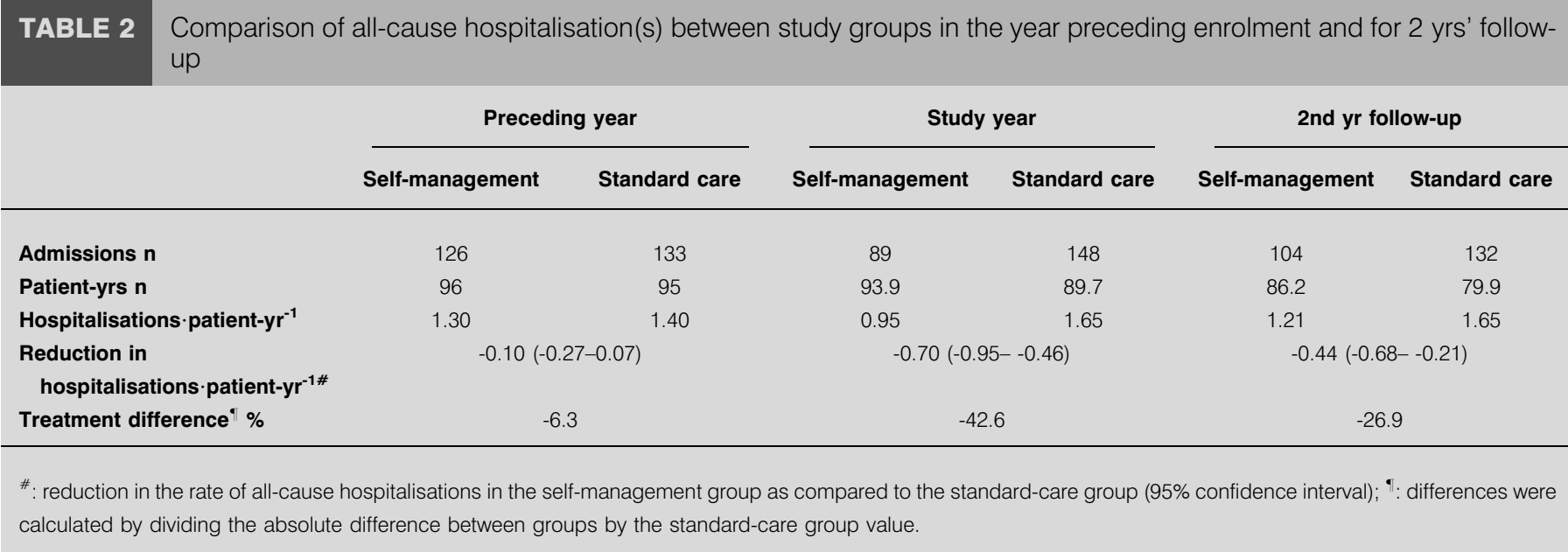




\begin{tabular}{|c|c|c|c|c|c|c|c|}
\hline \multirow[t]{3}{*}{ TABLE 3} & \multicolumn{7}{|c|}{$\begin{array}{l}\text { Comparison of all-cause emergency room (ER) visits between study groups in the year preceding enrolment and for } \\
2 \text { yrs' follow-up }\end{array}$} \\
\hline & & \multicolumn{2}{|c|}{ Preceding year } & \multicolumn{2}{|c|}{ Study year } & \multicolumn{2}{|c|}{ 2nd yr follow-up } \\
\hline & & Self-management & Standard care & Self-management & Standard care & Self-management & Standard care \\
\hline ER visits $n$ & & 256 & 268 & 211 & 317 & 217 & 255 \\
\hline ER visits $\cdot p$ & ent-yr ${ }^{-1}$ & 2.7 & 2.8 & 2.2 & 3.5 & 2.5 & 3.2 \\
\hline \multicolumn{2}{|c|}{ Reduction in ER visits patient-yr ${ }^{-1 \#}$} & \multicolumn{2}{|c|}{$-0.1(-0.21-0.02)$} & \multicolumn{2}{|c|}{$-1.3(-1.18--1.42)$} & \multicolumn{2}{|c|}{$-0.7(-0.58--0.82)$} \\
\hline Treatment & ference $\%$ & \multicolumn{2}{|c|}{-5.5} & \multicolumn{2}{|c|}{-36.5} & \multicolumn{2}{|c|}{-21.1} \\
\hline
\end{tabular}

\begin{tabular}{lcc} 
TABLE 4 & $\begin{array}{l}\text { Predictive factors of all-cause hospitalisations } \\
\text { during the 1st and 2nd yrs of follow-up after } \\
\text { adjustment for the intervention }\end{array}$ \\
\hline Variable & Adjusted OR & $\mathbf{9 5 \%} \mathbf{~ C l}$ \\
\hline Age $^{\#}$ & 1.05 & $1.03-1.06$ \\
Sex $^{\mathbf{S}^{*}}$ & 0.69 & $0.55-0.86$ \\
$\mathbf{S G R Q}^{+}$ & 1.015 & $1.008-1.022$ \\
$\mathbf{6 - m i n}^{+}$walk test $^{\mathbf{5}}$ & 0.998 & $0.997-0.999$ \\
Education level $^{+}$ & 0.81 & $0.73-0.90$ \\
\hline
\end{tabular}

OR: odds ratio; $\mathrm{Cl}$ : confidence interval. \#: age is treated as a continuous variable (per increased year); ${ }^{\bullet}$ : difference for female when compared to male; ${ }^{+}$: difference per one-point increase (reduced health status) using the $\mathrm{St}$ George's Respiratory Questionnaire (SGRQ); ${ }^{\text {s. }}$ 6-min walk test is given per increased metre on walking distance; ${ }^{f}$ : education level is treated as a fivecategory variable (from high to low)

\section{DISCUSSION}

It was shown recently that patients with moderate-to-severe COPD, who had received disease-specific self-management education with supervision for $1 \mathrm{yr}$, had fewer hospitalisations, emergency department visits and unscheduled physician visits as compared to patients on standard care [8].

The present study is the first to show that self-management education can reduce hospitalisation rates and emergency room visits over a 2 -yr follow-up period in patients with moderate-to-severe COPD. These sustained benefits to the healthcare system could potentially add to patients' quality of life by reducing institutionalisation. It is well known that COPD exacerbations and hospitalisations are determinants of poor quality of life and important cost drivers $[2,5,9]$.

The study also showed that older age and reduced health status are predictors of increased hospitalisations, while sex (female), increased walking capacity and higher education are predictors of reduced hospitalisations. Considering the limited resources of most healthcare systems, these findings may be of great practical value to help the clinician plan the management and follow-up of patients with COPD. This information might allow the design of future self-management programmes better adapted to the patient and more effective interventions.

In the present randomised clinical trial, treatment effects were not explained by differences in subjects' baseline characteristics. Subjects in the two study groups were similar with respect to sex, age, smoking, airflow limitation (FEV1 of $1.0 \mathrm{~L}$ ), disability (dyspnoea of 5/5 on the Medical Research Council scale for $50 \%$ of the patients) and health status (St George's Respiratory Questionnaire total score averaging 55). Dispensed medications were similar (results not provided) and all had similar access to healthcare and medications. The primary outcome, all-cause hospitalisations, was assessed using the provincial hospital database. Since this database includes all hospitalisations in the Province of Québec, it is unlikely to have underestimated hospitalisations in either study group.

It was not possible to blind participants as to treatment in this study. However, outcomes were assessed by an independent observer without knowledge of the intervention. Hospitalisation decisions were made by treating and/or emergency room physicians as part of usual healthcare and did not involve study investigators.

One potential limitation of the study is that during the 2nd yr of follow-up, continuous self-management education was left to the discretion of each participating centre and was not under strict protocol regulation as in the 1st yr. After completion of the clinical trial at $1 \mathrm{yr}$, only two participating centres were able to implement self-management into standard care for their COPD patients. In these two centres, but not in the other five participating centres, subjects had continuous access to a case manager. Any bias resulting from exposure of the standardcare subjects to the self-management intervention would have led to underestimation of its true effect. The fact that the benefits are sustained despite minimal or no sustained education after the 1st yr of the intervention suggests that patients in the self-management group have retained behaviours learned from the self-management programme.

People participating in this study were volunteers. This is not unique to self-management studies. As in the present study, previously published studies involving self-management have found that $\sim 50 \%$ of eligible out-patients participate [10]. 
Judgements about the appropriateness of generalising from participants to the population of interest can be made based on relevant patient characteristics. Patients had a range of airway obstructions, which, in most cases, were moderate to severe and associated with impaired health. However, all had previously experienced at least one hospitalisation for COPD.

In conclusion, this study has demonstrated that chronic obstructive pulmonary disease patient care integrating multicomponent self-management education, an action plan, supervision and communication with a case manager offers sustained benefits in reducing hospitalisations and emergency room visits. This approach should be part of standard care, with special attention given to chronic obstructive pulmonary disease patients with impaired health and frequent exacerbations.

\section{ACKNOWLEDGEMENTS}

The authors would like to thank the Régie de l'Assurance Maladie du Québec (RAMQ) for their services and technical assistance with the data collection. Last, but not least, many thanks to L. Auclair for her secretarial assistance and to S. Suissa and M. Edwardes from the Clinical Epidemiology Dept of the Royal Victoria Hospital (Montreal, Canada) for their statistical supervision and helpful orientation of analysis.

The authors are indebted to the COPD Axis Group of the Health Respiratory Network of the FRSQ, as well as to all COPD patients.

\section{REFERENCES}

1 Murray CJ, Lopez AD. Alternative projections of mortality and disability by cause 1990-2020: Global Burden of Disease Study. Lancet 1997; 349: 1498-1504.
2 Wouters EFM. Economic analysis of the confronting COPD survey: an overview of results. Respir Med 2003; 97: Suppl. C, S3-S14.

3 Ferrer M, Alonso J, Morra J, et al. Chronic obstructive pulmonary disease stage and health-related quality of life. Ann Intern Med 1997; 127: 1072-1079.

4 Osman LM, Godden DJ, Friend JAR, et al. Quality of life and hospital re-admission in patients with chronic obstructive pulmonary disease. Thorax 1997; 52: 67-71.

5 Seemungal TA, Donaldson GC, Paul EA, Bestall JC, Jeffries DJ, Wedzicha JA. Effects of exacerbation on quality of life in patients with chronic obstructive pulmonary disease. Am J Respir Crit Care Med 1998; 157: 1418-1422.

6 Ruchlin HS, Dasbach EJ. An economic overview of chronic obstructive pulmonary disease. Pharmacoeconomics 2001; 19: 623-642.

7 Collet J-P, Shapiro S, Ernst P, et al. Effects of an immunostimulating agent on acute exacerbations and hospitalizations in patients with chronic obstructive pulmonary disease. Am J Respir Crit Care Med 1997; 1456: 1719-1724.

8 Bourbeau J, Julien M, Maltais F, et al. Reduction of hospital utilization in patients with chronic obstructive pulmonary disease: a disease specific self-management intervention. Arch Intern Med 2003; 163: 585-591.

9 Jansson SA, Anderson F, Borg S, Ericsson A, Jonsson E, Lundback B. Costs of COPD in Sweden according to disease severity. Chest 2002; 122: 1994-2002.

10 Toobert D, Stycker L, Glasgow R, et al. If you build it, will they come? Reach and Adoption associated with a comprehensive lifestyle management program for women with type 2 diabetes. Patient Educ Couns 2002; 48: 99-105. 